\title{
Diferencias en satisfacción y estrés laboral entre trabajadores por cuenta ajena y autónomos
}

Antonia M. Antequera García, Alicia Cano Martín, María Olivares Peña, Inmaculada Pareja García, Alba Rodríguez González y Andrés Segura Alemany. Universidad de Granada

\author{
Recepción: 20 de mayo de 2016 | Revisión: 21 de mayo de 2016 | Aceptado: 30 de mayo de 2016 \\ Correspondencia: antoniaantequera@correo.ugr.es \\ Citar: Antequera, A.M., Cano, A., Olivares, M., Pareja, I., Rodríguez, A. y Segura, A. (2016). Diferencias en satisfacción y estrés \\ laboral entre trabajadores por cuenta ajena y autónomos. ReiDoCrea, 5(2), 7-13.
}

\begin{abstract}
Resumen: En esta investigación se ha pretendido buscar las posibles diferencias existentes en la satisfacción y el estrés laboral entre los trabajadores autónomos y los trabajadores por cuenta ajena. Para llevar esto a cabo se han utilizado dos cuestionarios a partir de los cuales se han obtenido los datos para su posterior análisis. A la vista de los resultados, se ha hallado una correlación negativa entre la satisfacción y el estrés laboral. Sin embargo, no se han encontrado evidencias de que los trabajadores autónomos tengan mayores niveles de satisfacción laboral y menores de estrés en el trabajo.
\end{abstract}

Palabras clave: Satisfacción laboral | Estrés laboral

Differences in Job Satisfaction and Stress among Self-employed People and Employees

Abstract: The aim of this research study was to determine the possible existence of differences in job satisfaction and stress among self-employed people and employees. In order to do so two questionnaires were used from which we obtained all the data for the subsequent analysis. In the light of the results a negative correlation between the two aforementioned variables was found. However, no evidence was found concerning self-employed people having higher job satisfaction levels and lower levels of stress.

Key words: Job satisfaction | Work stress

\section{Introducción}

El estudio de la satisfacción laboral tiene su origen en el análisis de las relaciones humanas, el cual defiende la relación positiva entre la satisfacción laboral y los resultados del trabajo (Bruce y Nyland, 2011; Mayo, 1949). Según Oplatka y Mimon (2008) la satisfacción laboral puede definirse como las actitudes y sentimientos de los individuos acerca de sus puestos de trabajo, favorables o no. Ésta se asocia positivamente con la percepción de autonomía en el ambiente de trabajo.

La autonomía es la percepción de la voluntad y el locus interno de causalidad (e.g., Aube et al. 2007; Mathieu y Zajac 1990; Riketta 2002), siendo una de las necesidades más importantes de satisfacer. La percepción de autonomía en el contexto social incrementa la motivación de los empleados y facilita el funcionamiento efectivo (Chang, Leach, y Anderman, 2015). Por lo tanto, un aumento en la satisfacción laboral podría disminuir los niveles de estrés de los trabajadores.

El estrés es un proceso integrado por variables tanto ambientales como individuales, que conlleva la percepción de una situación como amenazante (Lazarus y Folkman, 1984). En las últimas décadas, la cultura occidental ha experimentado un cambio en el ámbito laboral que lleva a una disminución de las exigencias físicas en el trabajo y un aumento de las demandas cognitivas e intelectuales (Artazcoza, Escribà-Agüirb y Cortès, 2006) con el consecuente aumento del estrés laboral. Karasek (1990) propone el Modelo de exigencias-control-apoyo, según el cual los estresores laborales son: sobrecarga de exigencias psicológicas, escaso control laboral y falta de apoyo del resto de compañeros, así como de los superiores. 
Como se ha señalado anteriormente, la percepción de autonomía por parte de los trabajadores produce en ellos efectos positivos, independientemente de si trabajan para otra persona o si lo hacen para sí mismos (Hackman y Oldham, 1975). Esto queda reflejado en estudios como el de Kinicki et al., (2002), en el que hallaron que una mayor percepción de autonomía en el trabajo se relaciona de forma positiva con mayor satisfacción laboral. De igual forma, Prottas (2008) respalda los mismos resultados en su estudio. A su vez este último encuentra una correlación negativa entre satisfacción laboral y el estrés en el trabajo. Por todo esto, las organizaciones pretenden llevar a cabo gestiones menos jerarquizadas para permitir así un mayor reparto de responsabilidades y autonomía a los trabajadores (Artazcoza et al., 2006).

Distintos estudios han encontrado diferencias en satisfacción laboral y estrés entre trabajadores autónomos y empleados. Por ejemplo, en 2008 Andersson encontró evidencia a favor de una mayor satisfacción laboral entre los trabajadores autónomos. Estos mismos resultados fueron observados en el estudio de Benz y Frey en 2008.

A la vista de esta información, en esta investigación se ha considerado que los trabajadores autónomos presentan un mayor nivel de autonomía, ya que disponen de una mayor flexibilidad horaria y una mayor libertad en la toma de decisiones que los empleados. Además, no mantienen ningún tipo de relación de subordinación, al contrario que los trabajadores por cuenta ajena.

El objetivo de esta investigación será comparar las diferencias en los distintos niveles de satisfacción y estrés laboral entre trabajadores autónomos y empleados. De acuerdo con la literatura previa sobre este tema, se espera que el estrés en el trabajo mantenga una correlación negativa con la satisfacción laboral. Además, se infiere que los trabajadores por cuenta propia, debido a su mayor nivel de autonomía, presentarán más satisfacción laboral y menos estrés en el trabajo que los trabajadores empleados.

\section{Método}

\section{Participantes}

En esta investigación han participado un total de 62 sujetos, de los cuales 31 eran trabajadores autónomos (con negocio propio) y los otros 31 eran trabajadores por cuenta ajena. Además, 30 de ellos eran hombres y 32 eran mujeres. Las edades de los sujetos oscilaban entre 23 y 63 años, con una media de edad de 44,048 años. Todos ellos eran de nacionalidad española y residentes en la provincia de Granada, España. Para su participación en el experimento, se fue visitando distintos locales solicitando así su colaboración. En este proceso, se les informó del anonimato de su participación así como de su total libertad de abandono del estudio si lo estimaran oportuno.

\section{Materiales}

Para la realización de este estudio se han utilizado dos cuestionarios en formato de papel:

1. Cuestionario de Satisfacción Laboral S20/23 (Meliá y Peiró, 1998): se trata de un cuestionario destinado a adultos que permite evaluar la satisfacción laboral considerando las variables motivacionales y temporales a las que están expuestos los trabajadores. Está compuesto por 23 ítems con un formato de respuesta tipo Likert que oscila entre 1 y 7 (de Muy Insatisfecho a Muy Satisfecho). Además consta de un total de diez preguntas sobre Datos Descriptivos de los participantes. 
El cuestionario muestra un alpha de Cronbach de 0.92 , oscilando sus factores entre 0.76 y 0.89 . Cuenta además con un total de cinco dimensiones: Satisfacción con la Supervisión, Satisfacción con el Ambiente Físico de trabajo, Satisfacción con las Prestaciones recibidas, Satisfacción Intrínseca del trabajo y Satisfacción con la Participación.

2. Cuestionario de estrés laboral Job Stress Survey (JSS) (Spielberger y Vagg, 1999). Su traducción y adaptación al español fue realizada por Ibermutua y TEA Ediciones (2010). Se trata de un cuestionario destinado a la población laboral a partir de los 18 años que permite evaluar la severidad percibida y la frecuencia de ocurrencia de 30 situaciones estresantes a las que se exponen habitualmente los trabajadores. Consta de 60 ítems con un formato de respuesta tipo Likert que oscila entre 1 y 9 (de bajo a alto) en el caso de la Severidad Percibida, y de 0 a 9+ (número de días) en el caso de la Frecuencia de Aparición en los últimos 6 meses. Este cuestionario, presenta un alpha de Cronbach de 0.80 . El JSS está formado por un total de dos escalas: JS-S Severidad del Estrés Laboral y JS-F Frecuencia del Estrés Laboral.

\section{Procedimiento}

En este estudio se ha utilizado un diseño de encuesta transversal, caracterizado por la recogida de datos a través de los cuestionarios anteriormente explicados en una única muestra y en un único momento temporal, concretamente en el periodo transcurrido entre el 16 y el 29 de Noviembre de 2015. Las Variables Dependientes evaluadas han sido la Satisfacción y el Estrés Laboral. Por otro lado, la Variable Independiente manipulada ha sido el tipo de empleo (autónomo/contratado).

En primer lugar, los sujetos firmaron un consentimiento informado en el que daban su aprobación para la participación en la investigación. Tras esto, a cada participante se le entregó una copia de los cuestionarios con sus respectivas condiciones y explicaciones. La única labor de los sujetos consistía en cumplimentarlos y devolvérselos a los experimentadores.

Para el análisis de los resultados obtenidos y presentados a continuación, ha sido utilizado el programa estadístico informático IBM-SPSS.

\section{Resultados}

Para llevar a cabo este análisis de resultados se han realizado las medias de todas las dimensiones de los cuestionarios y las correlaciones existentes entre estrés y satisfacción laboral de cada nivel, utilizando la correlación de Pearson, teniendo en cuenta que $p<.05$.

Tras realizar el análisis de correlación (Tabla 1) se ha encontrado una relación negativamente significativa entre la intensidad del estrés con la satisfacción intrínseca así como con el ambiente físico. Lo mismo ocurre con la frecuencia del estrés, la cual correlaciona negativa y significativamente con la satisfacción sobre la supervisión, la satisfacción con el ambiente físico y la satisfacción con las prestaciones. 


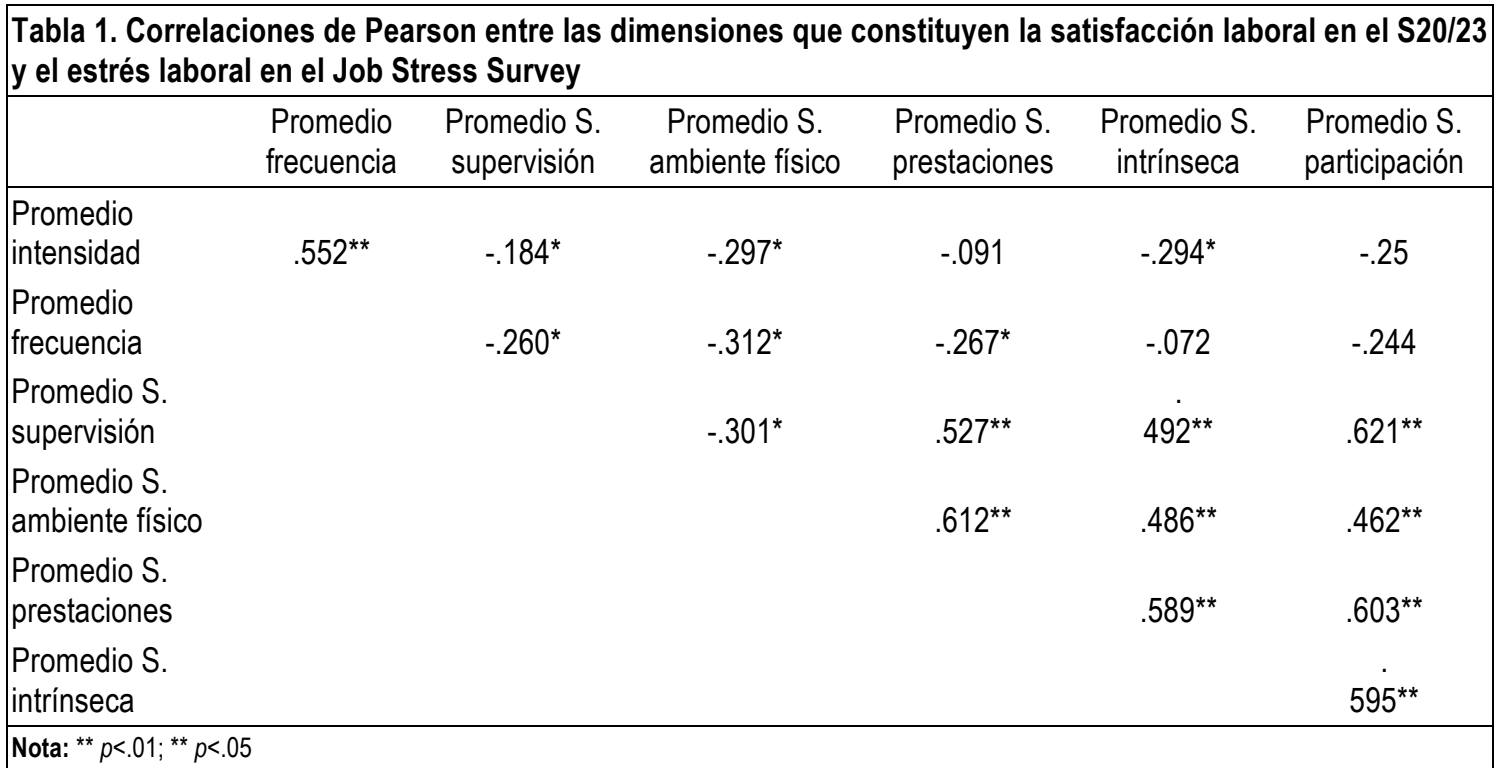

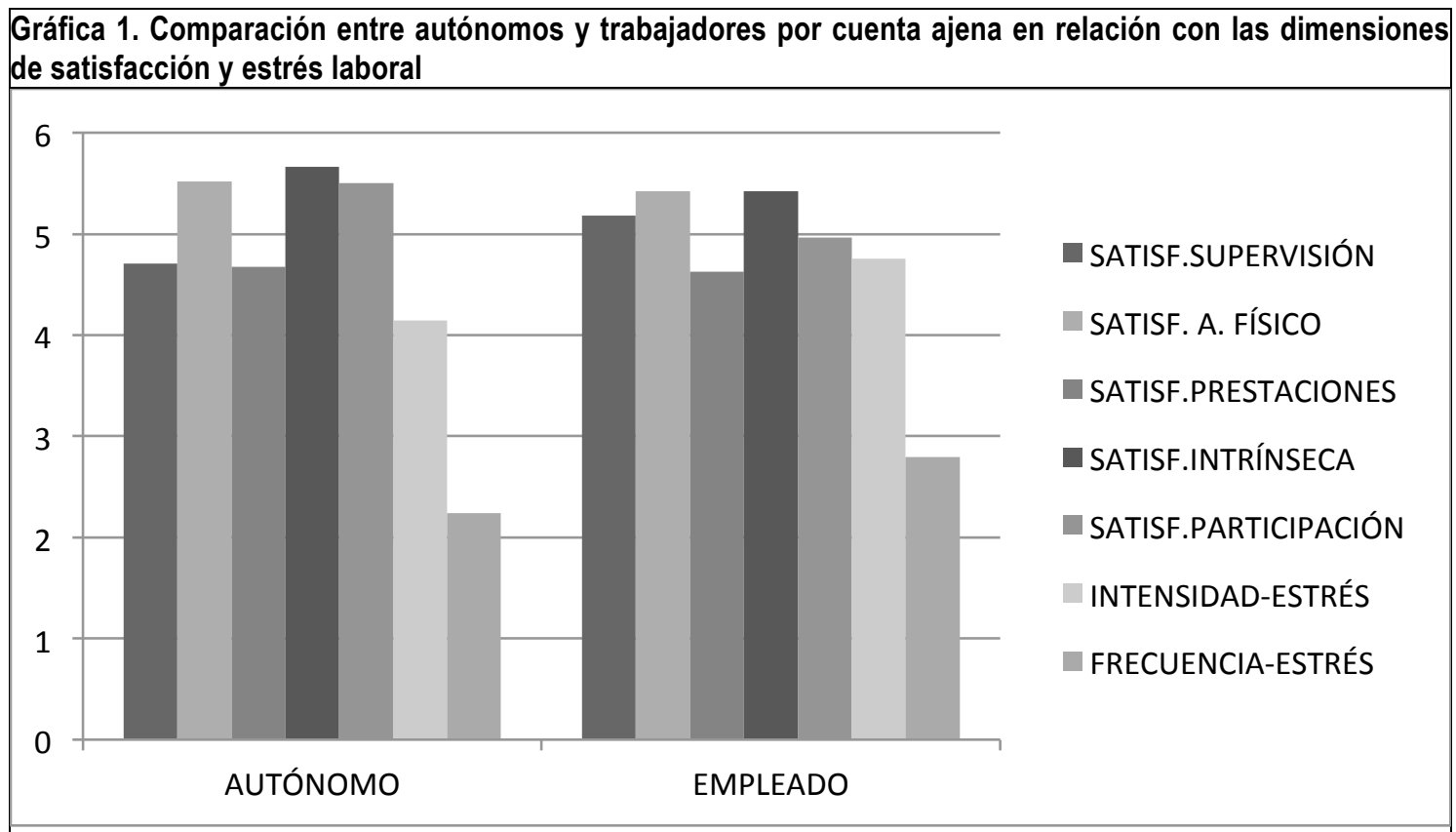

Para comparar las medias obtenidas en las diferentes dimensiones de ambos cuestionarios se realizó una T-Student para muestras independientes, a partir de la cual se obtuvieron los siguientes resultados:

No se encontraron diferencias significativas entre autónomos y empleados en las dimensiones del JSS de intensidad de estrés $\left(t_{(60)}=-1.542, p<.05\right)$ y de frecuencia del estrés $\left(t_{(60)}=-1.438, p<.05\right)$. De igual modo, tampoco hubo diferencias significativas entre ambos grupos en las dimensiones del S20/23 de satisfacción con la supervisión $\left(t_{(60)}=-1.619, p<.05\right)$, satisfacción con el ambiente físico $\left(t_{(60)}=.355, p<.05\right)$, satisfacción con las prestaciones $\left(t_{(60)}=.198, p<.05\right)$, satisfacción con la participación $\left(t_{(60)}=1.747\right.$, $p<.05)$ y satisfacción intrínseca $\left(t_{(60)}=1.073, p<.05\right)$. A pesar de la inexistencia de relaciones significativas, merece la pena destacar unas medias superiores de los trabajadores por cuenta ajena en las dimensiones de estrés laboral y, contrariamente, unas medias superiores de los trabajadores autónomos en la mayoría de las dimensiones de satisfacción laboral, exceptuando la de satisfacción con las prestaciones y la de satisfacción con la supervisión. 
Por último, durante el análisis de los datos se han utilizado además, a modo de exploración, otras variables sociodemográficas (Tabla 2). Así, se ha analizado el emolumento mensual, la edad, los años laborales, la situación laboral, el tipo de horario, las horas laborales semanales y las categorías jerárquicas de cada uno de los sujetos en su trabajo. A raíz de estos análisis se han encontrado relaciones significativas entre el salario mensual y la satisfacción con las prestaciones laborales, es decir, un mayor salario se traduce en mejores prestaciones laborales. También se han encontrado relaciones significativas entre la situación laboral de los trabajadores y su satisfacción laboral en cuatro dimensiones diferentes, las cuales son: satisfacción del ambiente físico, satisfacción en participación y en mayor medida, satisfacción intrínseca así como satisfacción de las prestaciones de trabajo. Por último, la categoría jerarquía que ocupa cada uno de los sujetos en su trabajo se ha relacionado de forma positiva con la satisfacción intrínseca, así como con la satisfacción en participación.

\begin{tabular}{|c|c|c|c|c|c|c|c|c|c|c|c|c|c|}
\hline & Edad & Pr. I. & Pr. F. & Pr. S. & Pr. A.F. & Pr.P. & Pr. Intr. & Pr. Par. & A.L. & S.L. & T.H. & H.S. & C.J. \\
\hline Salario & .132 & .176 & .140 & .104 & .186 & $.366^{\star \star}$ & .249 & .184 & .238 & $.299^{*}$ & -.082 & .200 & $.254^{*}$ \\
\hline Edad & & .208 & .114 & -.129 & -.054 & -.183 & -.079 & -.132 & $.723^{\star *}$ & $.260^{*}$ & .080 & $-.298^{*}$ & -.031 \\
\hline Pr. I. & & & $.552^{\star *}$ & -.184 & $-.297^{\star}$ & -.091 & $-.294^{*}$ & -.250 & .232 & -.177 & .069 & -.137 & -.123 \\
\hline Pr. F. & & & & $-.260^{*}$ & $-.312^{*}$ & $-.267^{*}$ & -.072 & -.244 & .165 & -.133 & -.065 & -.085 & -.018 \\
\hline Pr. S. & & & & & $.301^{*}$ & $.527^{* *}$ & $.490^{* *}$ & $.621^{* *}$ & -.107 & .235 & .045 & -.197 & -100 \\
\hline Pr. A.F. & & & & & & $.612^{* *}$ & $.486^{* *}$ & $.462^{* *}$ & -.235 & $.262^{*}$ & -.058 & .160 & .162 \\
\hline Pr. P. & & & & & & & $.589^{* *}$ & $.603^{* *}$ & -.079 & $.368^{* *}$ & -.023 & .149 & .163 \\
\hline Pr. Intr. & & & & & & & & $.595^{* *}$ & -.045 & $.470^{* *}$ & -.211 & .075 & $.355^{\star *}$ \\
\hline Pr. Par. & & & & & & & & & -193 & $.257^{\star}$ & -.120 & .036 & $.296^{\star}$ \\
\hline A.L. & & & & & & & & & & $.326^{* *}$ & .009 & -.075 & -.042 \\
\hline S.L. & & & & & & & & & & & .010 & .073 & $.332^{* *}$ \\
\hline T.H. & & & & & & & & & & & & -.084 & -.209 \\
\hline H.S. & & & & & & & & & & & & & $.429^{* *}$ \\
\hline \multicolumn{14}{|c|}{ Nota: *valores significativos $p<.01 ;{ }^{* *}$ valores muy significativos $p<.05$} \\
\hline ral; & & & & & & & Par. & & & & & & ;ituación \\
\hline
\end{tabular}

\section{Discusión}

A la vista de los resultados obtenidos se han encontrado las siguientes conclusiones. Respecto a la primera hipótesis, la cual suponía una correlación negativa entre la satisfacción y el estrés laboral, los datos hallados en los análisis realizados parecen apoyar la idea de Prottas (2008), al encontrarse una relación significativamente negativa entre las dimensiones de estrés y las de satisfacción laboral. Esta relación concuerda con la información reflejada en la literatura previa sobre este tema, la cual defiende que una mayor percepción de satisfacción laboral se relacionará con una menor percepción de estrés en el trabajo, permitiendo así una calidad de vida laboral mucho más positiva y eficiente.

En lo referente a la segunda hipótesis, la cual defendía la idea de que los trabajadores autónomos tendrían mayores niveles de satisfacción laboral y menores niveles de estrés en el trabajo en comparación con los trabajadores por cuenta ajena, como bien 
explicaban Andersson (2008) y Benz y Frey (2008) en sus respectivos estudios, no se han hallado diferencias significativas entre las dimensiones de ambas variables y el tipo de trabajo. En consonancia con Hackman y Oldham (1975), los sujetos se benefician de autonomía en el trabajo, independientemente de si trabajan para sí mismos o para otra persona. Sin embargo, y contrariamente a lo esperado según numerosas asociaciones entre la autonomía y el trabajo por cuenta propia, no existe evidencia de que los trabajadores autónomos valoren o se beneficien más de la autonomía que los trabajadores por cuenta ajena. Esto parece demostrar que el tipo de trabajo no influye ni en la satisfacción ni en el estrés laboral. Respecto a las variables sociodemográficas analizadas para intentar dar una explicación a los resultados obtenidos, se ha encontrado la existencia de relaciones significativas. Estas relaciones muestran que quizás los niveles de satisfacción laboral dependan de variables como, la situación laboral en mayor medida, seguida de la categoría jerárquica en el trabajo y por último el salario, con independencia de que el trabajador sea autónomo o empleado.

Desde una perspectiva práctica, los resultados obtenidos en esta investigación son instructivos para las personas en términos de orientación profesional. De este modo, incluso aunque los trabajadores autónomos no valoren la autonomía en el trabajo más de lo que lo hacen los trabajadores tradicionales, el trabajo por cuenta propia, ya sea como propietarios de pequeñas empresas que emplean a otros o como contratistas independientes, aparece como una forma viable de que las personas mejoren la calidad de sus vidas de trabajo, aumentando así su autonomía y satisfacción laboral (Kinicki et al., 2002).

Es importante destacar también algunas limitaciones metodológicas y procedimentales que podrían haber sesgado los resultados encontrados. En este línea, hay que mencionar la escasa cantidad de la muestra (únicamente 62 sujetos), las posibles diferencias individuales entre los participantes y el breve periodo de tiempo que se ha tenido para pasar los cuestionarios. Es necesario comentar también que algunos de los trabajadores autónomos de la muestra tuvieron complicaciones a la hora de rellenar el cuestionario de estrés laboral JSS, por lo que fue necesario aportarles instrucciones adicionales. Esta falta de comprensión en el momento de contestar podría haber producido sesgos en sus respuestas.

Por último, para la realización de investigaciones futuras se propone corregir estos errores metodológicos y, además del tipo de trabajo, estudiar la posible relación de otras variables, como el sexo o la personalidad. Así, hombres y mujeres podrían mostrar preferencias distintas hacia un tipo de trabajo u otro, pudiendo dar lugar a diferencias en sus niveles de satisfacción y estrés laboral. Otra variable que podría tenerse en cuenta a la hora de llevar a cabo un estudio similar sería el que trabajadores por cuenta ajena y trabajadores por cuenta propia pertenecieran a un mismo campo laboral, pues quizás de este modo las diferencias entre ambos grupos de estudio sean más significativas y sustanciales al existir de manera más clara y evidente diferencias en las tareas y funciones que realizan autónomos y empleados. De igual modo, el perfil de personalidad de las personas también podría actuar como variable mediadora entre el tipo de trabajo y la satisfacción y el estrés, pudiendo tener las personas con distintos rasgos unas preferencias específicas.

\section{Referencias}

Alexopoulos, E.C., Palatsidi, V., Tigani, X., \& Darviri, C. (2014). Exploring Stress Levels, Job Satisfaction, and Quality of Life in a Sample of Police Officers in Greece. Safety and health at work, 5(4), 210-215.

Araujo, B.L., Segovia, A.O. y Silla, J.M.P. (2007). El papel modulador de la implicación con el trabajo en la relación entre el estrés y la satisfacción laboral. Psicothema, 19(1), 81-87. 
Artazcoz, L., Escribà-Agüir, V. y Cortes, I. (2006). El estrés en una sociedad instalada en el cambio. Gaceta Sanitaria, 20, 71-78.

Aube, C., Rousseau, V., \& Morin, E.M. (2007). Perceived Organizational Support and Organizational Commitment: The Moderating Effect of Locus of Control and Work Autonomy. Journal of Managerial Psychology, 229, 479-495.

Bruce, K. and Nyland, C. (2011). Elton Mayo and the Deification of Human Relations. Organization Studies, 32(3), 383405.

Chang, Y., Leach, N., \& Anderman, E.M. (2015). The role of perceived autonomy support in principals' affective organizational commitment and job satisfaction. Social Psychology of Education, 1-22.

Esteva, M., Larraz, C. y Jiménez, F. (2006). La salud mental en los médicos de familia: efectos de la satisfacción y el estrés en el trabajo. Revista clinica española, 206(2), 77-83.

Hackman, J.R., \& Oldham, G.R. (1975). Development of the Job Diagnostic Survey. Journal of Applied Psychology, 60(2), 159-70.

Kautonen, T., Hytti, U., Bögenhold, D., \& Heinonen, J. (2012). Job satisfaction and retirement age intentions in Finland: Self-employed versus salary earners. International Journal of Manpower, 33(4), 424-440.

Kinicki, A.J., McKee-Ryan, F.M., Schriesheim, C.A., \& Carson, K.P. (2002). Assessing the construct validity of the Job Descriptive Index: a review and meta-analysis. Journal of Applied Psychology, 87(1), 14-32.

Lazarus, R.S., \& Folkman, S. (1984). Stress, appraisal and doping. Nueva York: Springer.

Mathieu, J.E., \& Zajac, D.M. (1990). A review and meta-analysis of the antecedents, correlates, and consequences of organizational commitment. Psychological Bulletin, 108, 171-194.

Oplatka, I., \& Mimon, R. (2008). Women principals' conceptions of job satisfaction and dissatisfaction: An alternative view? International Journal of Leadership in Education, 11(2), 135-153.

Prottas, D. (2008). Do the self-employed value autonomy more than employees? Research across four samples. Career Development International, 13(1), 33-45. 